with an emphasis on anthropometrical measures as a determinant for athletic prowess and selection, were found. For activities and participations, the 'educative' benefits of physical exercise for the formation of psychological factors such as character, resilience and morality were described. For the influence of contextual factors, such as attitudes and cultural influences, Britain saw the pursuit of a 'selected' ancient Greek ideal of combined intellectual and physical development, with an emphasis on all round activities and 'moderation'. For the USA, 'hard' and sometimes specialised physical training was deemed as important for the development of 'manly' virtues. For both countries the detrimental effect overtraining for boys was recognised. Female exercise was promoted in separate learning environments and with activities of a lower intensity than for boys. However, research insights from biomedical sciences such as physiology and neuroscience did not systematically inform physical education practices in Britain and the USA, despite the growth of inter-disciplinary groups and the increasing availability of biomedical publications in the LVA.

Conclusion The ICF-CY supports the historiography of physical education for children and youth during the late Victorian era. This has a parallel with the modern era of health promotion and the knowledge gained from sport and exercise science.

\section{G441 HISTORY OF PAEDIATRIC NEPHROLOGY IN EUROPE - 50 YEARS OF HOPE}

${ }^{1,2}{ }^{2}$ Tse, ${ }^{2,3} \mathrm{H}$ Maxwell, ${ }^{2,4} \mathrm{AR}$ Watson, ${ }^{2,5} \mathrm{E}$ Levtchenko, ${ }^{2,6} \mathrm{M}$ Christian. 'Department of Paediatric Nephrology, Great North Children's Hospital, Newcastle Upon Tyne, UK; ${ }^{2}$ On behalf of European Society for Paediatric Nephrology; ${ }^{3}$ Department of Paediatric Nephrology, Royal Hospital for Sick Children, Glasgow, UK; ${ }^{4}$ University of Nottingham, Nottingham, UK; ${ }^{5}$ Department of Paediatric Nephrology, University Hospital of Leuven, Leuven, Belgium; ' Department of Paediatric Nephrology, Nottingham Children's Hospital, Nottingham, UK

\subsection{6/archdischild-2018-rcpch.430}

2017 marked the 50th anniversary of the European Society for Paediatric Nephrology (ESPN) foundation meeting in Glasgow. It was also the centenary of the first ever kidney biopsy in a child or adult also in Glasgow. Since then tremendous advances in medical, nursing and psycho-social care have been made.

As part of the anniversary we held and recorded on film a history symposium delivered by early pioneers. Topics included the start of dialysis and transplantation in children, use of renal biopsy to in nephrotic syndrome management, collaborative work of the ESPN and development of the multi-disciplinary team. Creating this opportunity for retired experts to reflect on 50 years of progress gives a unique wide-angled perspective. These journeys of discoveries challenges current thinking and can act like a calibrating device to ensure good past ideas are not lost.

We also created an oral history video repository (www.youtube.com/c/ESPNhistory) where 35 early pioneers recorded their stories for (P)ity. Their voices recount vividly the desperation families and children suffered when treatment was ineffective and transplant or dialysis unfathomable - followed by the era of rapid innovation. We learn from the pioneers their own personal stories of self-sacrifice, heroism and dogged persistence. Of interest to our current generation is the historical and technological context: the Iron Curtain curtailing travel, no internet, emails or Medline. These pioneers matured research from personal case series to the international collaborations we have today.

Storing oral history is important as most of this rich history was never written up, remembered only in anecdotes. With today's inexpensive technology it is simple and we achieved breath by collaborating internationally. We owe it to future generations to collate a living oral history of medicine of this remarkable bygone age from those who were there. The use of cheap smartphone filming and a customised repository channel on internet video social media opens up new ways to connect history of medicine to future generations.

\section{G442(P) THERAPEUTIC CLOWNING - HISTORY, MEDICINE AND EVIDENCE}

${ }^{1} \mathrm{~F}$ Finlay, ${ }^{2} \mathrm{~S}$ Lenton, ${ }^{3} \mathrm{~A}$ Baverstock. ${ }^{1}$ Child Health, Virgin Care, Bath, UK; ${ }^{2}$ Royal United Hospitals, Bath, UK; ${ }^{3}$ Paediatrics, Musgrove Park Hospital, Taunton, UK

\subsection{6/archdischild-2018-rcpch.431}

History of clowning There is documented evidence for the role of a 'Jester' figure in most ancient cultures including, Egyptian, Greek, Chinese, Roman and mediaeval Europe. The term 'clown', derived from the Scandinavian word 'cloyne' to describe a 'clumsy person', started to appear in the 1500 s to describe foolish characters in Shakespearean plays.

Modern clowns are strongly associated with the tradition of the circus clown which developed from earlier comedic theatre roles during the 19th centuries. Joseph Grimaldi (17781837) being the first recognisable ancestor of the modern clown.

Clowns in hospitals/health environments

Dr Hunter Doherty 'Patch' Adams was the founder of clown doctors. He put on a red nose as he worked in hospitals believing that humour and laughter creates an atmosphere of trust and love between staff and patients. Clown doctors now work throughout the world including the Big Apple Circus Clown Care Unit, in USA, the Theodora Foundation in Europe, Africa and Asia and the Humour Foundation Clown Doctor Program in Australia.

Therapeutic clowns are selected for personal qualities such as communication skills, compassion and empathy so they complement clinicians and play an integral role in the paediatric care team using magic, music and storytelling to engage children and enable them to deal with the emotions they may experience while in hospital such as fear, anxiety, boredom and loneliness.

Evidence of effect Clowns may have positive cognitive, physiological, social and emotional effects. A review of the paediatric literature reveals their value in the following areas:

- Reducing stress and anxiety during the pre-operative period, in both children and parents

- Reducing pain during invasive medical procedures

- Reducing anxiety during sexual abuse examination

- Shorter induction time for anaesthesia and faster discharge

- Promoting patient- and family-centred care in PICU

- Positive impact on staff working in disaster area field hospital

- Improving team function 